method of coming at a rough-and-ready conclusion regarding the size of men, than the one which I have adopted.

This subject has interested me from the standpoint of better nutrition. It will be observed that the American man is decidedly gaining in size and weight. If this has happened during twenty years of the American frying-pan, dyspeptic bread, pale pie, and cooking in general under the supervision of cooks who were sent from the wrong place where the meat did not come from, what may be expected when the American woman learns how to cook? Cannot some one obtain data for comparison with these sizes from the statistics of military recruits and conscripts in Europe, or from the contractors for army clothing ?

Boston, Nov. 3.

Edward AtKinson.

\section{The Sense of Smell.}

IT is quite customary, when treating of the senses, to speak slightingly of smell and taste, as if they were of little importance in the economy of life. When the subject of training the senses is under consideration, little is ever said of training the nose, while much space is devoted to educating the eye, the ear, and the hand. It is certainly true that smell does not rank with sight and hearing, and demands less care, perhaps, for its cultivation ; and yet it plays an important rôle, and should receive its due share of attention in any scheme of education.

The function of smell is fourfold. Like the higher senses, it belongs to the intellectual endowments. It is a part of the mind. Through it the mind is reached, roused, and quickened. The percepts and concepts gained through the sense of smell can be named, described, analyzed, compared, and classified. They may thus become the means of a good degree of intellectual life. Smell is a source of knowledge. Through it the mind discerns those qualities in things which we denominate odor. This knowledge it can obtain in no other way. A surprisingly large number of objects have their own peculiar odor. The onion, the carrot, the beet, and all other vegetables have characteristic odors; so have fruits, flowers, spices, and many gases, as well as animals, meats, etc. The knowledge of the kind, quality, and condition of things that can be obtained by the sense of smell, is very extensive. Not only the druggist, the chemist, the cook, but others likewise, make much practical use of the nose as a source of knowledge, having its own special scientific interest. But smell does a highly important work in enabling us to detect foul, hurtful odors. The nose is placed at the entrance to the mouth as a sentinel to guard it from receiving unwholesome food. It is the watch-dog of the stomach. A fourth, scarcely less important function of smell is that of giving pleasure. The nose is capable of ministering to our happiness even more, perhaps, than the touch or the taste. One with a cultivated nose has delights that another knows not.

There is even a greater need for some systematic training of the sense of smell than of the so-called higher senses. The ordinary experiences of life and the regular work of the school-room necessarily give to the eye, ear, and mind considerable exercise; while the smell is called into use much less frequently out of school, and scarcely at all in school. Besides, the words expressive of smell percepts and concepts are far less numerous and exact than corresponding words for sight and hearing; so that the training incident to the use of language is likely to be far less extensive and accurate in the case of the nose than in that of the eye, ear, and hand. Add to this the low estimate generally placed upon the sense of smell, and the popular indifference to its training, as shown in the fact, that, while we have elaborate schemes for training the eye in knowledge of form and color, we have practically none for training the nose in the performance of its proper functions, and we may challenge for this useful member the sympathy and interest due to neglected merit and overlooked modesty.

In every primary school there should be some special attention paid to the education of this sense. This should aim to secure, first, the frequent exercise of the sense until it acquires strength proportionate to its duties. It should not be overworked, nor called into undue prominence, but should receive its proper share of attention till it acquires both strength and sensitiveness. 'Second, the training should be such as to develop a high power of discrimi- nation, so that the pupil can discern quickly and accurately the different odors that are presented. Third, the growth in discriminative power should be accompanied pari passu with language. Each distinct odor should be named, and the closest association should be created between the idea and the word, so that the one shall recall the other. The pupil should be exercised in analyzing complex odors, so as to be able to detect the presence of different substances in the same compound. He should be instructed in noxious smells, which indicate the presence of harmful substances, and should have some knowledge of the disagreeable odors, their origin, and the method of their removal.

Boys might receive a little special training as a preparation for laboratory or scientific work, and girls be instructed in view of their possible duties as cooks or housekeepers. A few very simple principles suffice for suggesting a plan of carrying this scheme into effect. The work should be begun in the primary grades. This is a period of sense-activity, when the child is being aroused to mental life through sense-perception, acquainting itself with the sensequalities of the universe, and storing up ideas for future use. If the senses are neglected at this period, the opportunity for training them may never return. At first the work should be simple, making very light demands upon the sense. A few common fruits, flowers, and spices or gums may be used, with a view of forming a sharp discrimination, quick recognition, and accurate naming. The drill exercises should be very brief, aiming at thoroughness rather than multiplicity, and may be alternated with lessons in form, color, place, number, etc. The lessons should be graded so as to increase in difficulty, and should be so systematized as to secure the fourfold end of varied activity, knowledge, health, and pleasure. Each step forward in sense-discrimination should be accompanied with drill in oral and written language. For ordinary purposes it will be sufficient to make the child well acquainted with perhaps one hundred distinct odors, separate and in combination; and these for the most part should be of those things a knowledge of which will be of most service in daily life.

When the sense has been properly trained in childhood, and a habit of wise use established, the pupil will be able to call it into exercise on all needful occasions, and, on the basis of this general culture, can, if need be, secure a highly specialized development of the sense, meeting all the requirements of extraordinary occasions.

Providence, R.I., Nov. 3.

THOMAS J. MORGAN.

\section{Answers.}

I 5. IS THE TRUMPET-CREEPER POISONOUS?-The belief is general in many parts of the South and South-west that both the trumpet-creeper and the Virginia creeper (Ampelopsis quinquefolia) are poisonous. I have always acted upon the opinion that this belief is as unfounded in the one case as in the other. A little experience of the poisonous Rhus will make an ignorant person afraid of every vine found growing in the woods.

JOHN C. BRANNER.

Little Rock, Ark., Oct. 3r.

I5. Is THE TRUMPeT-CReeper PoIsonous? - No. This is Tecoma radicans, and climbs trees, posts, walls, etc., by means of thousands of rootlets. It is trained around many verandas and about door-yards for ornament. No one was ever poisoned by it. It has been often mistaken for Rhus radicans of Linnæus (the climbing variety of $R$. toxicodendron), which also climbs by means of thousands of rootlets. The stems of the two clinging to trees resemble each other very much. I have had many cases of Rhuspoisoning, but never heard of any thing being poisoned by the $T e$ coma. Many times when persons have exclaimed in alarm, "That is poison-vine, don't touch it!" I have, to their consternation, seized the leaves of Tecoma, rubbed them over my face and hands, and even chewed them. With sixty years of daily intimacy with these plants, I feel justified in these statements. D. L. PHARES. Agricultural College P.O., Miss., Nov. 5.

16. The Archbald pot-holes were described by Dr. John C. Branner in the Proceedings of the American Philosophical Society, vol. xxiii., pp. 353-357 (read Feb. 19, I 887), and Mr. Charles A. Ashburner in the 'Annual Report of the Pennsylvania Geological Survey for I 885,' pp. 61 5-626.

Cornell Univ., Ithaca, N.Y., Nov. 7. 